\title{
Interplay between heart rate and its variability: a prognostic game
}

\author{
Jerzy Sacha* \\ Department of Cardiology, Regional Medical Center, Opole, Poland \\ *Correspondence: sacha@op.pl \\ Edited by: \\ Gaetano Santulli, "Federico II" University Hospital, Italy and New York Presbyterian Hospital - Manhattan, USA \\ Reviewed by: \\ Yu-Chieh Tzeng, University of Otago, New Zealand \\ Paolo Allegrini, Consiglio Nazionale delle Ricerche, Italy
}

Keywords: heart rate, heart rate variability, heart rate dynamics, prediction, correlation, risk factor

In the last decades, heart rate (HR) and heart rate variability (HRV) were extensively investigated in various clinical and laboratory settings and proved to be significant risk factors for different outcomes and patients' populations (Kannel et al., 1987; Bravi et al., 2011; Antoni et al., 2012). During that time, a number of new methods permitting to explore different aspects of HR variability and dynamics have been implemented (Task Force of the European Society of Cardiology, and the North American Society of Pacing and Electrophysiology, 1996; Schmidt et al., 1999; Bauer et al., 2006). However, most of them yield parameters which are essentially associated with HR (Tsuji et al., 1996; Cygankiewicz et al., 2004; Sacha and Pluta, 2005, 2008; Lewek et al., 2009; Sacha, 2013), therefore, they actually carry information not only on the variability but also on HR itself (Sacha, 2014a,b). In fact it is hard to ascertain which of these two (i.e., HR or variability) really matters in the prognostic significance of HRV (Sacha, 2013, 2014a,b). Recently, a new approach which enables to weaken (or even eliminate) or strengthen the association between $\mathrm{HR}$ and its variability has been proposed (Sacha et al., 2013a). The principles of this method are simple, i.e., by division of RR interval tachograms by the corresponding average $\mathrm{RR}$ intervals, the variability of RR intervals of slow HR is attenuated, but that of fast $\mathrm{HR}$ is relatively amplified and the resulting HRV loses its correlation with HR. On the other hand, by multiplication of RR interval tachograms by their average RR intervals, the variability of slow HR is boosted but that of fast HR is relatively suppressed, and consequently, the correlation between HRV and HR is growing. By division or multiplication by higher powers of average RR intervals stronger effect on the HRV/HR relationship can be achieved (Sacha et al., 2013a). Moreover, by division by very high powers, this relationship may even be inverted, i.e., from negative to positive one (Figure 1A) (Sacha et al., 2013b).

Recent studies with implementation of this method have shown that HR may have different impact on the prognostic ability of HRV for different outcomes (Sacha et al., 2013c). In general, it seems that for populations and events where HR is a significant risk factor the enhancement of its impact improves the prognostic value of HRV, however, for groups and outcomes where HR is not or is a weak risk factor, the exclusion of its influence increases the HRV prediction capacity (Sacha et al., 2013c, 2014; Sacha, 2014a,b). In particular, such phenomena were observed in the study addressing HRV and HR in different genders after myocardial infarction (Sacha et al., 2014). In other words, HR was a strong risk factor of cardiac death in men and strengthening its influence on HRV boosted the HRV prediction performance for cardiac mortality, conversely, HR was a poor predictor of non-cardiac death in male subgroup and weakening its impact augmented the HRV prognostic value for noncardiac mortality. However, HR did not predict any outcomes in females and the exclusion of its influence improved the HRV prognostic ability for every mode of death in women (Figure 1B) (Sacha et al., 2014).
The concept of such prognostic HRV and HR interaction has been recently confirmed in a large group of patients undergoing exercise tests (i.e., 1288 participants) (Pradhapan et al., 2014). The study showed that HR right before exercise was not a risk factor of death and elimination of its influence improved the predictive capability of the respective HRV, conversely, HR during recovery phase was a significant mortality predictor and the enhancement of its impact augmented the respective HRV prognostic performance (Pradhapan et al., 2014).

Low HRV and high HR are usually associated with worse prognosis, however, there are also situations where such a coincidence represent favorable prospect-this can be seen during exercises (Dewey et al., 2007). In the aforementioned study by Pradhapan et al., higher HRV and lower HR (i.e., below $125 \mathrm{bpm}$ ) during recovery phase were related with an increased risk of both cardiac and non-cardiac death (Pradhapan et al., 2014). However, if one inverts the HRV/HR relationship from negative to positive one (i.e., by division of HRV indices by high powers of their corresponding average RR intervals), higher HRV represents good prognosisFigure 1C (Pradhapan et al., 2014).

Thus, the interplay between HRV and HR turns out to be quite complicated. However, the unraveling of this remarkable game, by using the method of strengthening or weakening the HRV/HR dependence (Sacha et al., 2013a), may yield valuable prognostic information (Sacha et al., 2013c, 2014; Pradhapan et al., 2014; Sacha, 2014a,b). This is particularly important in women, in whom 


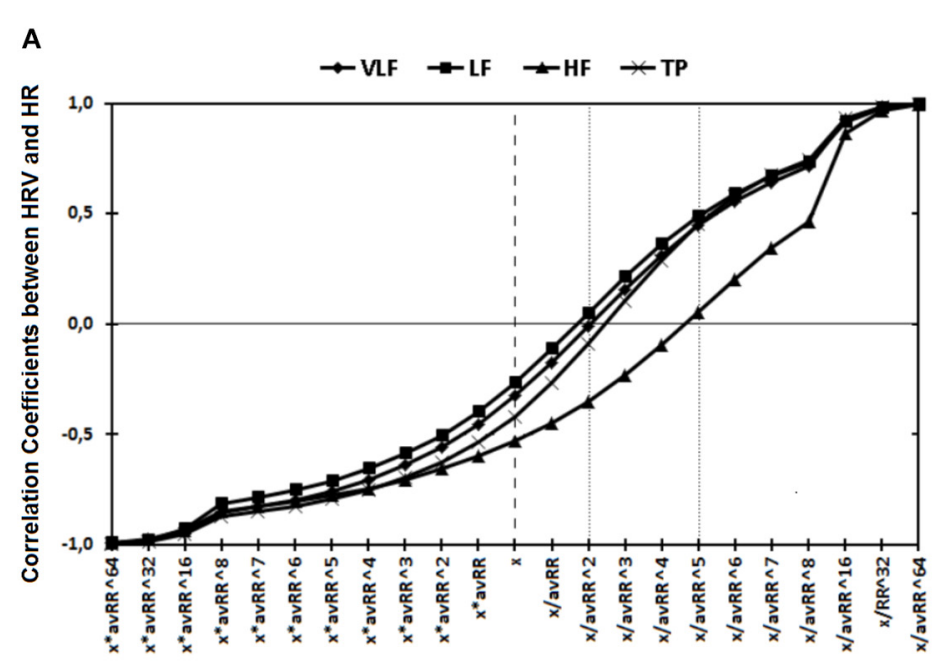

Modified HRV indices

-

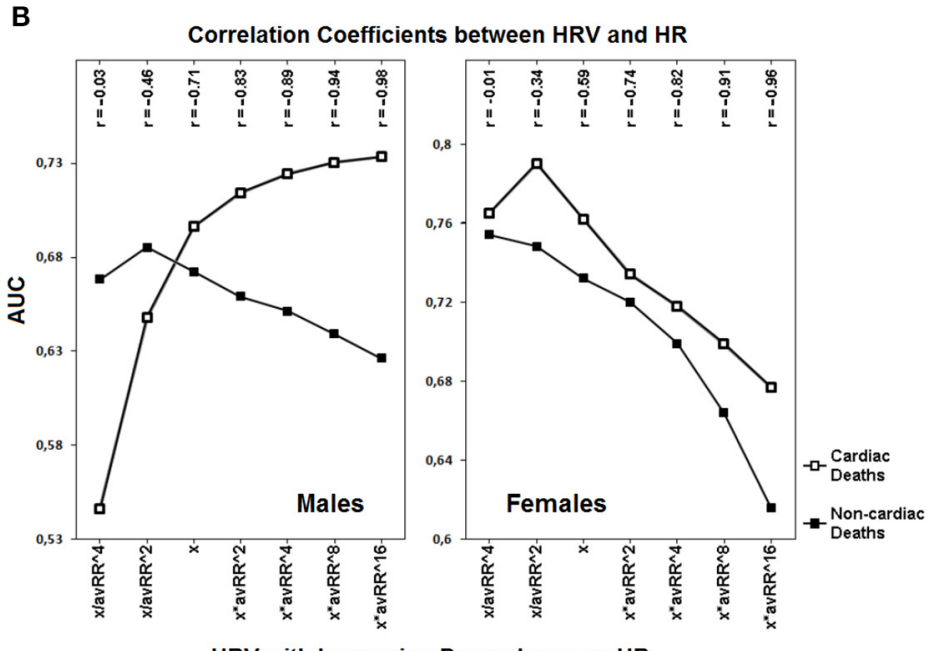

HRV with Increasing Dependence on HR

C

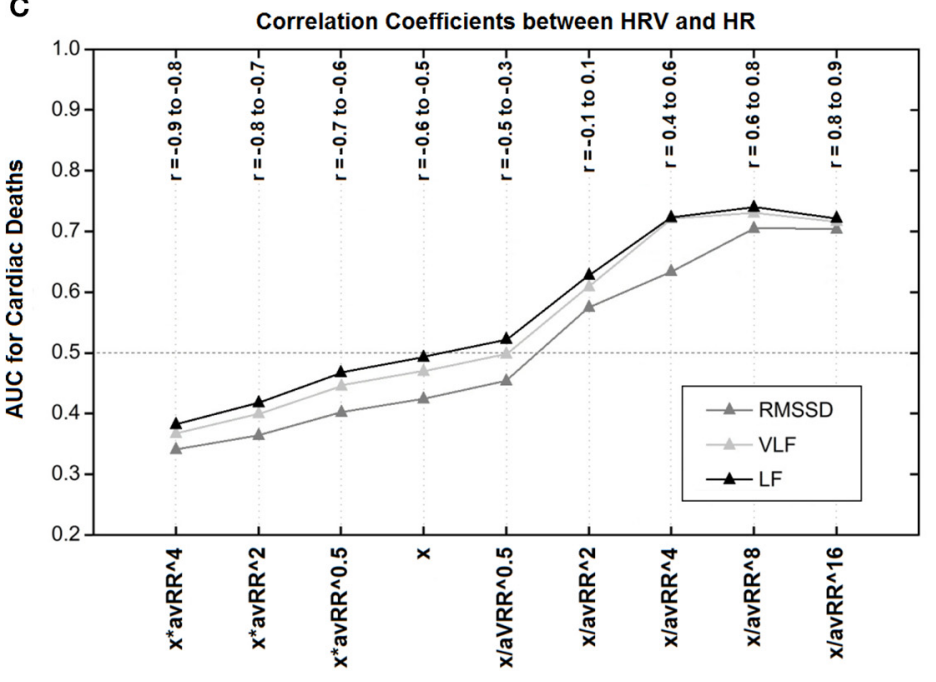

Modified HRV indices

FIGURE 1 | Continued 
FIGURE 1 | (A) Correlation coefficients between different modified spectral HRV indices and HR are presented. The " $x$ " denotes standard HRV indices which all are inversely associated with $H R$; multiplication by different powers of the corresponding average RR interval (avRR) increases this negative relationship (the left half of the diagram); but division by avRR to different powers diminishes the HRV dependence on HR and may even inverse this relationship, i.e., from negative to positive one (the right half of the diagram). Of note, to become HR-independent (i.e., to achieve insignificant correlation coefficients) VLF, LF and TP have to be divided by $\operatorname{avRR}^{\wedge} 2$, but $\mathrm{HF}$ by $a R^{\wedge} 5$ (see respective markers on dotted lines)-this is due to the fact that HF is initially more dependent on HR (compare respective markers on the dashed line). HF, high frequency component; LF, low frequency component; VLF, very low frequency component; TP, total power (Reprinted from Sacha et al., 2013b). (B) The prediction performance (i.e., AUC, area under receiver-operator characteristic curves) for different classes of modified HRV indices (i.e., very low frequency components of HRV spectrum) and their correlation coefficients ( $r$ ) with HR in males and females are depicted. The " $x$ " denotes standard HRV indices, other indices were calculated by division or multiplication of " $x$ " by different powers of the corresponding average $R R$ interval (avRR). In the first two classes (i.e., $x / a v R R^{\wedge} 4$ and $\left.x / a v R^{\wedge} 2\right)$, the $H R V$ dependence on $H R$ was weakened, while it was strengthened in the last four classes (i.e., $x^{*} \operatorname{avRR}^{\wedge} 2, x^{*} \operatorname{avRR} R^{\wedge} 4, x^{*} \operatorname{avRR}^{\wedge} 8$ and $x^{*} a v R R^{\wedge} 16$ ). As HRV is becoming more dependent on HR (i.e., from the first to the seventh class), its predictive ability increases in men for cardiac death but decreases for non-cardiac one, while in women, it decreases for both outcomes. It is noteworthy that HR was a strong predictor of cardiac death and a weak predictor of non-cardiac death in males, however, in females HR did not predict any mode of death (Reprinted with modification from Sacha et al., 2014). (C) Predictive performance for cardiac mortality (i.e., AUC, area under receiver-operator characteristics curves) for different classes of modified HRV indices and their correlation coefficients ( $r$ ) with $H R$, during the recovery after exercise test are presented. The " $x$ " denotes standard HRV indices, other indices were calculated by multiplication or division of " $x$ " by different powers of the corresponding average RR interval (avRR). The AUC $<0.5$ indicates that higher HRV is related with worse prognosis but AUC > 0.5 means that higher HRV is associated with better prognosis. Standard HRV indices (i.e., $x$ ) are negatively correlated with HR and after multiplication by different powers of avRR this negative correlation becomes tighter, along with the improvement in their predictive ability (i.e., AUC is getting lower and lower) - of note, higher values of these indices are related with worse prognosis. However, the division by different powers of avRR makes $H R V$ indices either independent on $H R$ (i.e., $x / a v R R^{\wedge} 2$ ) or positively correlated with $H R$ (i.e., $x / a v R R^{\wedge} 4, x / \operatorname{avRR} R^{\wedge} 8$ and $x / a v R R^{\wedge} 16$ ) along with the increase in their predictive power-higher values of these indices are associated with better prognosis (i.e., AUC > 0.5). LF, low frequency component; RMSSD, root mean square successive differences; VLF, very low frequency component (Reprinted with modification and permission from Pradhapan et al., 2014).
HR is a weak (or even is not) risk factor and actually can cover the prognostic value of HRV - yet, this detrimental effect may be eliminated by the removal of HRV dependence on HR (Sacha et al., 2014).

Currently, it is hard to conclude how to practically employ the aforementioned method in clinical settings. However, a concept of a separate approach to HR and its variability should enable us to determine which of the two quantities presents higher predictive performance for a given population and outcome. Probably, it will give us possibilities to increase the prognostic value of HRV by the suitable modification of its relationship with HR. It should be stressed that such a method may be employed to any other HR dynamics analysis which parameters are significantly correlated with HR (Sacha, 2014a,b). The first experiences with using this approach are encouraging, however, the concept requires further investigations in various clinical situations.

\section{REFERENCES}

Antoni, M. L., Boden, H., Delgado, V., Boersma, E., Fox, K., Schalij, M. J., et al. (2012). Relationship between discharge heart rate and mortality in patients after acute myocardial infarction treated with primary percutaneous coronary intervention. Eur. Heart J. 33, 96-102. doi: 10.1093/eurheartj/ ehr293

Bauer, A., Kantelhardt, J. W., Barthel, P., Schneider, R., Mäkikallio, T., Ulm, K., et al. (2006). Deceleration capacity of heart rate as a predictor of mortality after myocardial infarction: cohort study. Lancet
367, 1674-1681. doi: 10.1016/S0140-6736(06) 68735-7

Bravi, A., Longtin, A., and Seely, A. J. (2011). Review and classification of variability analysis techniques with clinical applications. Biomed. Eng. Online 10:90. doi: 10.1186/1475-925X-10-90

Cygankiewicz, I., Wranicz, J. K., Bolinska, H., Zaslonka, J., and Zareba, W. (2004). Relationship between heart rate turbulence and heart rate, heart rate variability, and number of ventricular premature beats in coronary patients. J. Cardiovasc. Electrophysiol. 15, 731-737. doi: 10.1046/j.15408167.2004.03613.x

Dewey, F. E., Freeman, J. V., Engel, G., Oviedo, R., Abrol, N., Ahmed, N., et al. (2007). Novel predictor of prognosis from exercise stress testing: heart rate variability response to the exercise treadmill test. Am. Heart J. 153, 281-288. doi: 10.1016/j.ahj. 2006.11.001

Kannel, W. B., Kannel, C., Paffenbarger, R. S. Jr., and Cupples, L. A. (1987). Heart rate and cardiovascular mortality: the Framingham Study. Am. Heart J. 113, 1489-1494. doi: 10.1016/0002-8703 (87)90666-1

Lewek, J., Wranicz, J. K., Guzik, P., Chudzik, M., Ruta, J., and Cygankiewicz, I. (2009). Clinical and electrocardiographic covariates of deceleration capacity in patients with ST-segment elevation myocardial infarction. Cardiol. J. 16, 528-534.

Pradhapan, P., Tarvainen, M. P., Nieminen, T., Lehtinen, R., Nikus, K., Lehtimäki, T., et al. (2014). Effect of heart rate correction on preand post-exercise heart rate variability to predict risk of mortality-an experimental study on the FINCAVAS cohort. Front. Physiol. 5:208. doi: 10.3389/fphys.2014.00208

Sacha, J. (2013). Why should one normalize heart rate variability with respect to average heart rate. Front. Physiol. 4:306. doi: 10.3389/fphys.2013.00306

Sacha, J. (2014a). Interaction between heart rate and heart rate variability. Ann. Noninvasive
Electrocardiol. 19, 207-216. doi: 10.1111/anec. 12148

Sacha, J. (2014b). Heart rate contribution to the clinical value of heart rate variability. Kardiol. Pol. doi: 10.5603/KP.a2014.0116. [Epub ahead of print].

Sacha, J., Barabach, S., Statkiewicz-Barabach, G., Sacha, K., Muller, A., Piskorski, J., et al. (2013a). How to strengthen or weaken the HRV dependence on heart rate-Description of the method and its perspectives. Int. J. Cardiol. 168, 1660-1663. doi: 10.1016/j.ijcard.2013.03.038

Sacha, J., Barabach, S., Statkiewicz-Barabach, G., Sacha, K., Muller, A., Piskorski, J., et al. (2013c). How to select patients who will not benefit from ICD therapy by using heart rate and its variability? Int. J. Cardiol. 168, 1655-1658. doi: 10.1016/j. ijcard.2013.03.040

Sacha, J., Barabach, S., Statkiewicz-Barabach, G., Sacha, K., Muller, A., Piskorski, J., et al. (2014). Gender differences in the interaction between heart rate and its variability - how to use it to improve the prognostic power of heart rate variability. Int. J. Cardiol. 171, e42-e45. doi: 10.1016/j. ijcard.2013.11.116

Sacha, J., and Pluta, W. (2005). Different methods of heart rate variability analysis reveal different correlations of heart rate variability spectrum with average heart rate. J. Electrocardiol. 38, 47-53. doi: 10.1016/j.jelectrocard.2004.09.015

Sacha, J., and Pluta, W. (2008). Alterations of an average heart rate change heart rate variability due to mathematical reasons. Int. J. Cardiol. 128, 444-447. doi: 10.1016/j.ijcard.2007. 06.047

Sacha, J., Sobon, J., Sacha, K., and Barabach, S. (2013b). Heart rate impact on the reproducibility of heart rate variability analysis. Int. J. Cardiol. 168, 4257-4259. doi: 10.1016/j.ijcard.2013. 04.160

Schmidt, G., Malik, M., Barthel, P., Schneider, R., Ulm, K., Rolnitzky, L., et al. (1999). Heart-rate turbulence after ventricular premature beats as 
a predictor of mortality after acute myocardial infarction. Lancet 353, 1390-1396. doi: 10.1016/ S0140-6736(98)08428-1

Task Force of the European Society of Cardiology, and the North American Society of Pacing and Electrophysiology, A. (1996). Heart rate variability: standards of measurement, physiological interpretation and clinical use. Circulation 93, 1043-1065. doi: 10.1161/01.CIR.93. 5.1043

Tsuji, H., Venditti, F. J. Jr., Manders, E. S., Evans, J. C., Larson, M. G., Feldman, C. L., et al. (1996). Determinants of heart rate variability. J. Am. Coll.
Cardiol. 28, 1539-1546. doi: 10.1016/S0735-1097 (96)00342-7

Conflict of Interest Statement: The author declares that the research was conducted in the absence of any commercial or financial relationships that could be construed as a potential conflict of interest.

Received: 31 July 2014; accepted: 23 August 2014; published online: 12 September 2014.

Citation: Sacha J (2014) Interplay between heart rate and its variability: a prognostic game. Front. Physiol. 5:347. doi: 10.3389/fphys.2014.00347
This article was submitted to Clinical and Translational Physiology, a section of the journal Frontiers in Physiology.

Copyright (c) 2014 Sacha. This is an open-access article distributed under the terms of the Creative Commons Attribution License (CC BY). The use, distribution or reproduction in other forums is permitted, provided the original author(s) or licensor are credited and that the original publication in this journal is cited, in accordance with accepted academic practice. No use, distribution or reproduction is permitted which does not comply with these terms. 